\title{
A NONINVASIVE TRANSFER SYSTEM FOR POLARIZED RENAL TUBULE EPITHELIAL CELL SHEETS USING TEMPERATURE-RESPONSIVE CULTURE DISHES
}

\author{
A. Kushida, M. Yamato, Y. Isoi, A. Kikuchi and T. Okano* \\ Institute of Advanced Biomedical Engineering and Science, Tokyo Women's Medical University, \\ 8-1 Kawada-cho, Shinjuku-ku, Tokyo 162-8666, Japan
}

\begin{abstract}
We used temperature-responsive culture dishes onto which the temperature-responsive polymer, poly $(\mathrm{N}-$ isopropylacrylamide), was covalently grafted for tissue engineering. Confluent cells harvested as intact sheets from these surfaces by simple temperature reduction can be transferred to various surfaces including additional culture dishes, other cell sheets, and tissues. In order to examine the maintenance of cell polarity, Madin-Darby canine kidney cells and human primary renal proximal tubule epithelial cells which had developed apical-basal cell polarity in culture, were subjected to cell sheet transfer. This functional and structural cell polarity, which is susceptible to treatment with trypsin, was examined by immunohistochemistry and transmission electron microscopy. Using our cell-sheet method, the noninvasive transfer of these cell sheets retaining typical distributions of $\mathrm{Na}^{+} / \mathrm{K}^{+}$-ATPase, GLUT-1, SGLT-1, aquaporin-1, neutral endopeptidase and dipeptidylendopeptidase IV, could be achieved. The transferred cell sheets also developed numerous microvilli and tight junctions at the apical and lateral membranes, respectively. For biochemical analysis, immunoblotting of occludin, a transmembrane protein that composes tight junctions, was conducted and results confirmed that occludin remained intact after cell sheet transfer. This two-dimensional cell sheet manipulation method promises to be useful for tissue engineering as well as in the investigation of epithelial cell polarity.
\end{abstract}

Key Words: Tissue engineering, $\operatorname{poly}(N-$ isopropylacrylamide), tight junction, cell sheet engineering, confocal laser scanning microscopy.

\footnotetext{
*Address for correspondence:

Teruo Okano

Institute of Advanced Biomedical Engineering and Science Tokyo Women's Medical University

8-1 Kawada-cho, Shinjuku-ku, Tokyo 162-8666, Japan

Telephone Number: +81-3-3353-8111 ext. 30233

FAX Number +81-3-3359-6046

E-mail: tokano@abmes.twmu.ac.jp
}

\section{Introduction}

The success of cell therapy is closely dependent on the development of cell culture techniques. While these methods have advanced significantly, there are still problems that need to be resolved before cell-based therapies can be effectively applied. One practical shortcoming is the inability to harvest intact cultured cells from culture dishes without loss of highly differentiated cell functions, as typical cell harvest techniques use proteolytic enzymes that irreversibly damage highly differentiated cells. For example, one of the most important properties of epithelial cells is the formation of a paracellular barrier to transport ions, proteins, and other molecules in the proper manner. Differentiated epithelial cells often develop tight junctions (TJs) forming an intracellular barrier to maintain their characteristic apicalbasal cell polarity. However, treatment with trypsin causes monolayer epithelial cells to dissociate into single cells and deprives them of their differentiated functions. Further culture is therefore required to recover the differentiated phenotype, because epithelial cells can develop apical-basal polarity only after the establishment of TJs between adjacent cells. We therefore applied our novel noninvasive cell manipulation method, using temperature-responsive culture dishes (Hirose et al., 2000), in order to determine whether these differentiated functions could be maintained.

The temperature-responsive polymer poly $(N$ isopropylacrylamide) (PIPAAm), which shows phase transition across its lower critical solution temperature (LCST) of $32^{\circ} \mathrm{C}$ (Heskins et al., 1968), is covalently grafted to tissue culture polystyrene (TCPS) surfaces by radical polymerization initiated with electron-beam irradiation (Yamada et al., 1990). These polymer-grafted culture surfaces exhibit a temperature-responsive property; that is, the surface is slightly hydrophobic under culture conditions at $37^{\circ} \mathrm{C}$ and reversibly changes to hydrophilic below the LCST. On temperature-responsive culture dishes, various cell types adhere, spread, and proliferate similarly to on nongrafted TCPS dishes at $37^{\circ} \mathrm{C}$. However, when the culture temperature is reduced below the LCST, the cells can be lifted from the surfaces without the need for trypsin or other degradative enzymes, because of the rapid hydration of PIPAAm (Okano et al., 1993). By using these temperature-responsive dishes we have developed a unique method to harvest and transfer cultured monolayer cell sheets from PIPAAm-grafted dishes to other surfaces, and designated it "twodimensional (2-D) cell sheet manipulation" (Hirose et al., 2000). Harvested cell sheets can readily adhere to other 
domains because extracellular matrix deposited during cell culture remains on the basal surfaces of cell sheets even after harvest (Kushida et al., 1999; Kushida et al., 2000; Yamato et al., 2001). Various cell sheets including endothelial cells (Hirose et al., 2000), Madin-Darby canine kidney (MDCK) cells (Kushida et al., 2001), keratinocytes (Yamato et al., 2001), cardiac myocytes (Shimizu et al., 2002), lung cells (Nandkumar et al., 2002), corneal epithelial cells (Nishida et al., 2004a), oral mucosal epithelial cells (Nishida et al., 2004b), and urothelial cells (Shiroyanagi et al., 2004) have been harvested and transferred with this method. These 2-D manipulated cell sheets are able to maintain cell viability, cell-to-cell junctions, and differentiated phenotypes. In the present study, MDCK cells, a canine renal tubule epithelial cell line, and primary human renal proximal tubule epithelial cells (RPTECs) were subjected to 2-D cell sheet manipulation and examined for the presence of TJs and the expression of an apical-basal polarity.

\section{Materials and Methods}

\section{Reagents}

$N$-Isopropylacrylamide (IPAAm) was kindly provided by Kohjin (Tokyo, Japan). The following materials were purchased from the respective companies. Trypsinethylenediaminetetraacetic acid (EDTA) solution, streptomycin, and penicillin were from Gibco BRL Life Technologies (Grand Island, NY, U.S.A); trypsin inhibitor (soybean type II-S), phenylmethylsulfonyl fluoride (PMSF), bovine serum albumin fraction V (BSA), and Tween 20 were from Sigma (St. Louis, MO, U.S.A); Dulbecco's modified Eagle's medium (DMEM) was from IWAKI glass (Chiba, Japan); fetal bovine serum (FBS) was from PAA Laboratories (Exton, PA, U.S.A.); protease inhibitor cocktail was from Wako (Osaka, Japan); rabbit anti-occludin polyclonal antibody was from Zymed Laboratories, Inc. (San Francisco, CA, U.S.A); ECL Western blotting detection reagents and horseradish peroxidase-conjugated donkey anti-rabbit immunoglobulins antibody were from Amersham (Buckinghamshire, U.K.); fluorescein isothiocyanate (FITC)-conjugated goat antibody against mouse IgG and FITC-conjugated goat antibody against rabbit immunoglobulins were from ICN Pharmaceuticals, Inc. Cappel Products (Aurora, OH, U.S.A.); rabbit anti-sodium glucose co-transporter-1 (SGLT-1) antibody, rabbit antiGLUT-1 antibody and rabbit anti-aquaporin 1 affinity purified polyclonal antibody were from CHEMICON International, Inc (Temecula, CA, U.S.A.); mouse antisheep $\mathrm{Na}^{+} / \mathrm{K}^{+}$-ATPase monoclonal antibody (M7-PB-E9) was from Affinity Bioreagents, Inc. (Golden, CO, U.S.A.); mouse anti-neutral endopeptidase (NEP) monoclonal antibody (ALB1) and mouse anti-dipeptidyl endopeptidase IV (DPP-IV) monoclonal antibody (BA5) were from Immunotech (Marseille, France); and rhodamineconjugated phalloidin was from Molecular Probes (Eugene, OR, U.S.A.).

\section{Preparation of temperature-responsive culture dishes}

Specific procedures for the preparation of PIPAAm-grafted cell culture dishes are described elsewhere (Hirose et al., 2000). Briefly, IPAAm monomer in 2-propanol solution was spread onto TCPS dishes (Falcon 3001, Becton Dickinson, Franklin Lakes, NJ, U.S.A.). The dishes were then subjected to electron beam irradiation with an Area Beam Electron Processing System (Nisshin High Voltage, Kyoto, Japan). The PIPAAm-grafted dishes were rinsed with cold distilled water to remove ungrafted IPAAm and sterilized by ethylene oxide gas. Untreated TCPS dishes were used as control surfaces.

\section{Cells and cell culture}

MDCK cells (passage 57) were provided by the Health Science Research Resources Bank (JCRB 9029, Osaka, Japan). MDCK cells were maintained in DMEM supplemented with $10 \%$ FBS, 100 units/mL penicillin, 100 $\mu \mathrm{g} / \mathrm{mL}$ streptomycin and used between passages 60 and 65 for all experiments. Primary human renal proximal tubule epithelial cells (RPTECs) were purchased from BioWhittaker, Inc. (Walkersville, MD, U.S.A.) and cultured in renal epithelial cell basal medium (REBM ${ }^{\mathrm{TM}}$, BioWhittaker) supplemented with $0.5 \% \mathrm{FBS}, 10 \mathrm{ng} / \mathrm{mL}$ human epidermal growth factor, $5 \mu \mathrm{g} / \mathrm{mL}$ insulin, $0.5 \mu \mathrm{g} /$ $\mathrm{mL}$ hydrocortisone, $0.5 \mu \mathrm{g} / \mathrm{mL}$ epinephrine, $6.5 \mathrm{ng} / \mathrm{mL}$ triiodothyronine, $10 \mu \mathrm{g} / \mathrm{mL}$ transferrin, $50 \mu \mathrm{g} / \mathrm{mL}$ gentamicin and $50 \mathrm{ng} / \mathrm{mL}$ amphotericin-B. Cells were maintained at $37^{\circ} \mathrm{C}$ in a humidified atmosphere of $5 \% \mathrm{CO}_{2}$. Cell morphology was monitored under a phase contrast microscope (ET300, Nikon, Tokyo, Japan).

\section{Two-dimensional cell-sheet manipulation}

MDCK cells or RPTECs were seeded on PIPAAm-grafted culture dishes at a cell density of $5 \times 10^{5}$ cells $/ 35 \mathrm{~mm}$ dish (about one-fourth confluent cell density) or $6.8 \times 10^{4}$ cells/ $35 \mathrm{~mm}$ dish (about one-eighth confluent cell density) respectively, and cultured at $37^{\circ} \mathrm{C}$. Three weeks after seeding, the dishes were transferred to an incubator set at $20^{\circ} \mathrm{C}$. After incubation for one hour, hydrophilic poly(vinylidene difluoride) (PVDF) membranes (Durapore membrane filter, pore size $5.0 \mu \mathrm{m}$, Millipore Corporation, Bedford, MA, U.S.A.) were placed into the culture dishes and the culture medium was aspirated such that the membranes attached to the cell layers. The membranes were then slowly peeled from the borders of the dishes with forceps and transferred to either porous polyethylene terephthalate (PET) membranes (Culture Insert ${ }^{\mathrm{TM}}$, Falcon 3090 , pore size $0.4 \mu \mathrm{m}$ ) or collagen-coated culture dishes. After the incubation at $20^{\circ} \mathrm{C}$ for 30 minutes, fresh culture medium was added. The PVDF membranes were then released from cell surfaces, allowing for simple removal using forceps.

\section{Immunoblotting}

Cultured MDCK cells were harvested by one of three different methods: trypsin-EDTA treatment, 2-D cell-sheet manipulation, or physical scraping with a rubber blade. For trypsin-EDTA treatment, MDCK cells were incubated 
with $0.25 \%$ trypsin- $0.02 \%$ EDTA (TE) in PBS at $37^{\circ} \mathrm{C}$ for 15 min. Harvested cells were washed three times with PBS containing protease inhibitors ( $0.25 \%$ trypsin inhibitor, 500 $\mu \mathrm{M}$ AEBSF, $150 \mathrm{nM}$ aprotinin, $1 \mu \mathrm{M}$ E-64, $500 \mu \mathrm{M}$ EDTA, $1 \mu \mathrm{M}$ leupeptin, and $1 \mathrm{mM}$ PMSF), and lysed in lysis buffer (20 mM Tris-buffered saline ( $\mathrm{pH} 7.4$ ) containing 1\% SDS, $2 \mathrm{M}$ Urea, and trypsin inhibitors). For 2-D cell-sheet manipulation, MDCK cells were recovered from the temperature-responsive surfaces with a hydrophilically modified PVDF membrane, after incubation at $20^{\circ} \mathrm{C}$ for 1 hour. After centrifugation at $1000 \times \mathrm{g}$ for $5 \mathrm{~min}$, the cell pellet was collected and dissolved in lysis buffer. For physical scraping, cells were removed from culture surfaces by a rubber blade in lysis buffer. Whole cell lysates were then electrophoresed on a $7.5 \%$ polyacrylamide gel with a $4.5 \%$ stacking gel in a discontinuous buffer system (Laemmli, 1970). An equal amount of protein was applied for each sample, as determined by the BCA protein assay (Pierce, Rockford, IL USA). Resolved proteins on the polyacrylamide gel were electronically transferred to a poly(vinylidene difluoride) transfer membrane (Immobilon$\mathrm{P}$, Millipore) (60 V for 3 hours). The membrane was incubated at $4{ }^{\circ} \mathrm{C}$ overnight in blocking solution which consisted of 5\% BSA in $20 \mathrm{mM}$ Tris-buffered saline $(\mathrm{pH}$ 7.4) containing $0.05 \%$ Tween 20 (TBS-Tween), and was then probed with rabbit anti-occludin polyclonal antibody (1:3000-dilution in TBS-Tween containing 1\% BSA) at room temperature for $90 \mathrm{~min}$. After extensive washing with TBSTween, the membrane was incubated with a 1:10000 dilution of horseradish peroxidase-conjugated donkey anti-rabbit Ig antibody at room temperature for $90 \mathrm{~min}$, and the bands representing occludins were detected by chemiluminescence of the peroxidase reaction using the ECL system.

\section{Fluorescence microscopy}

To prepare TE subcultured cells, cells were dissociated with TE treatment into single cell suspensions, replated under confluent conditions, and cultured for 5 hours. The 2-D manipulated and TE subcultured cells were fixed at room temperature with 4\% paraformaldehyde in PBS for $20 \mathrm{~min}$. Cells were washed with PBS and permeabilized with $0.5 \%$ Triton X-100 in PBS for 2 min. Cells were then blocked with $0.1 \% \mathrm{BSA}$ in PBS for 2 hours, and reacted with primary antibodies overnight at $4{ }^{\circ} \mathrm{C}$. Following three washes with $0.1 \%$ BSA in PBS, fixed cells were incubated with a 1:200 dilution of FITC-conjugated secondary antibodies for 1 hour and again washed three times. To examine F-actin, cells were co-stained with a 1:100 dilution of rhodamineconjugated phalloidin. Stained cells were observed under a microscope with fluorescence equipment (ET300, Nikon, Tokyo, Japan) and a confocal laser scanning microscope (TCS-SP, Leica Microsystems AG, Wetzlar, Germany). For transverse sections, images taken at $0.7 \mu \mathrm{m}$ steps in the $\mathrm{z}$ axis were projected through the z-axis and rebuilt as twodimensional tomography images with computer software. Cells stained without either primary or secondary antibody were used as negative controls. With negative control experiments, no fluorescence was detected by the conditions used for this study.

\section{Transmission electron microscopy}

MDCK cells cultured for three weeks were 2-D manipulated onto porous PET membranes by lowtemperature treatment and fixed with $4 \%$ glutaraldehyde in PBS at room temperature. Fixed samples were dehydrated through a graded alcohol series and embedded in Epok 812 (Oken, Tokyo, Japan). Ultrathin sections were cut with a diamond knife, and then stained with uranyl acetate and lead citrate. Samples were examined at $75 \mathrm{kV}$ under an electron microscope (JEM1010, JEOL, Tokyo, Japan).

\section{Results and Discussion}

MDCK cells and RPTECs were plated onto temperatureresponsive culture dishes grafted with PIPAAm. Both cell types adhered, spread, and proliferated to confluency on the grafted dishes, in the same manner as observed on nongrafted TCPS dishes. Apical-basal cell polarity is vital for the proper functions of renal tubule epithelial cells, such as reabsorption and this cell polarity is created and maintained by tight junctions (TJs) which are located at the most-apical region of the lateral membranes of epithelial cells. TJs function as a barrier between apical and basolateral plasma membrane domains, preventing the diffusion of solutes through paracellular pathways. TJs are also responsible for the ability of renal tubule epithelial cells to carry out vectorial ion transport as well as for creating polarized distributions of plasma membrane lipids, glycoproteins, and cytoskeletal proteins.

MDCK cells were plated at an initial cell density of approximately one-fourth confluency and harvested by physical scraping after various periods of cell culture. The expression of occludin, an integral membrane protein in $\mathrm{TJ}$ formation, was examined by immunoblotting (Fig. 1). In confluent MDCK cells, occludin is reported to be detected as multiple bands between 62 and $82 \mathrm{kDa}$ by SDS-PAGE analysis (Sakakibara et al., 1997). The higher molecular weight (MW) occludin bands are highly phosphorylated proteins, which are selectively concentrated at TJs, while non- or less- phosphorylated occludins are distributed along the basolateral membranes (Sakakibara et al., 1997). Highly phosphorylated occludin was detected three days after

\section{$1 d \quad 3 d \quad 1 w \quad 2 w \quad 3 w$

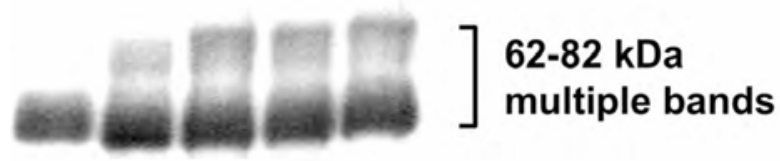

Fig. 1 Immunoblotting of MDCK cells with antioccludin antibody. MDCK cells were plated on PIPAAm-grafted culture dishes at an approximately one-fourth confluent cell density and cultured at $37^{\circ} \mathrm{C}$. At the indicated time periods, cells were recovered by physical scraping with a rubber blade and subjected to immunoblotting. 

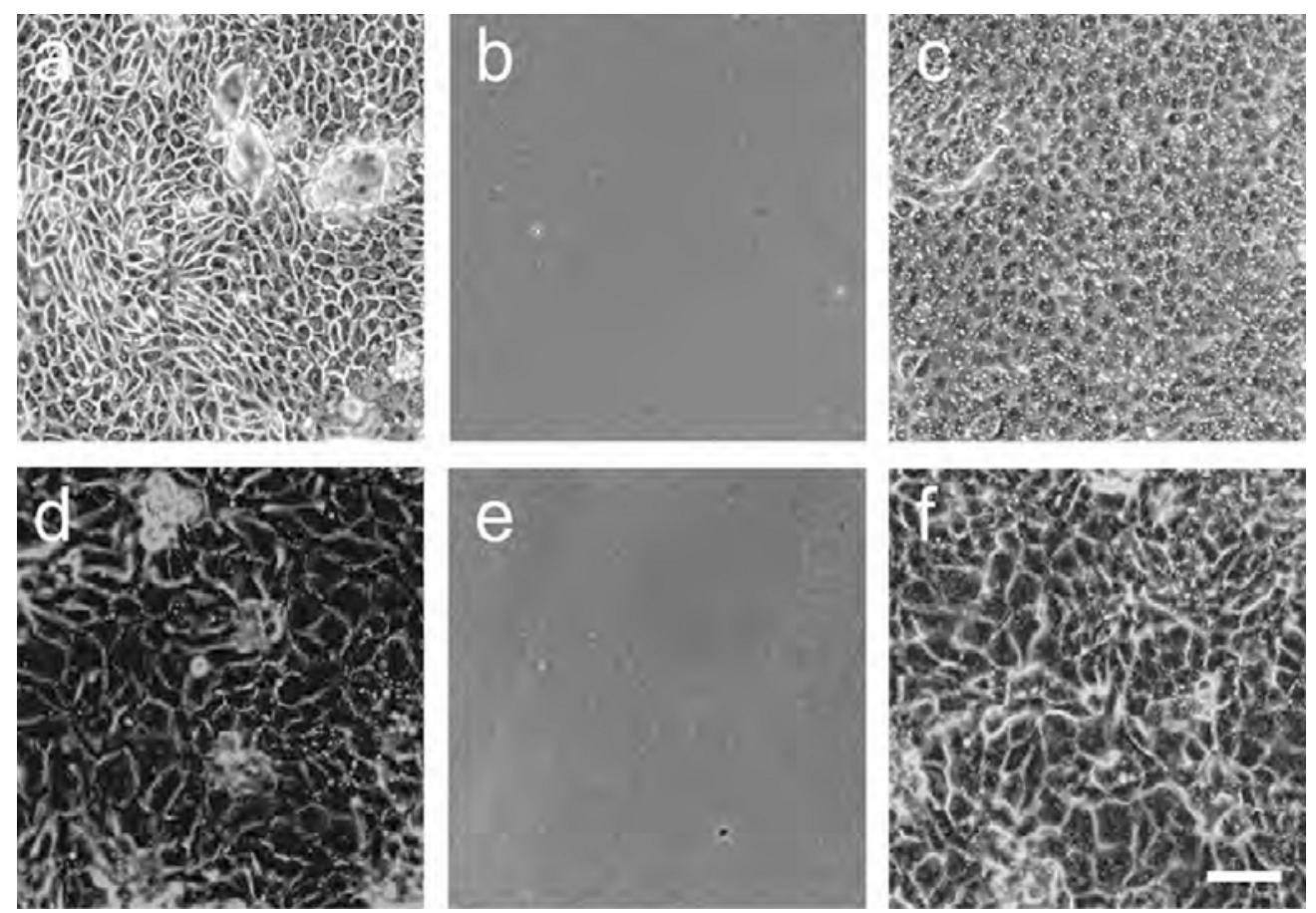

Fig. 2 Phase contrast microscopy of MDCK cells and RPTECs before and after two-dimensional manipulation. MDCK cells (upper) or RPTECs (lower) were plated on PIPAAm-grafted culture dishes and cultured at $37^{\circ} \mathrm{C}$ for three weeks $(a, d)$. Renal epithelial cells were subjected to low temperature treatment at $20^{\circ} \mathrm{C}$. These cells were completely harvested as a single contiguous cell sheet after one-hour incubation at $20^{\circ} \mathrm{C}$, and no cell remnants were observed on the PIPAAm-grafted dishes $(b, e)$. The harvested cell sheets were then transferred and readily adhered to other dishes after the 30 -min incubation at $20^{\circ} \mathrm{C}(c, f)$. Scale bar $=100 \mu \mathrm{m}$. Note that porous PET membranes slightly hampered the image contrast under a phase contrast microscope.
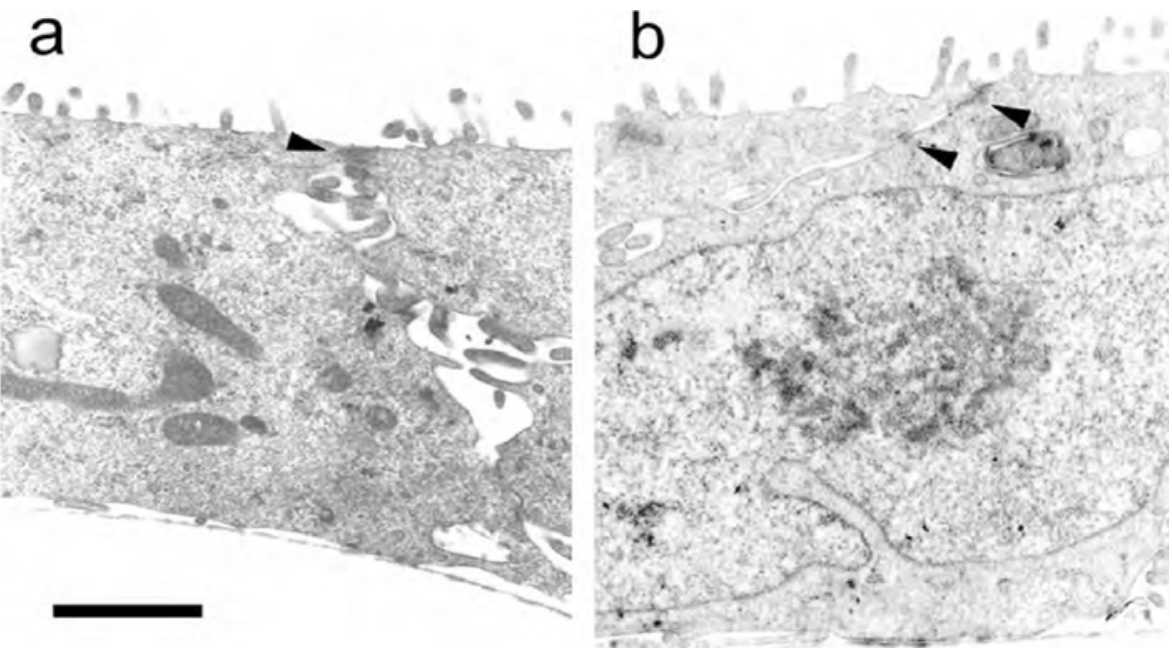

Fig. 3 Transmission electron microscopy of transferred MDCK cell sheets on Culture Insert ${ }^{\mathrm{TM}}$ membranes. Three-week cultured MDCK cells were subjected to 2-D cell sheet manipulation. Transferred MDCK cells were fixed immediately $(a)$ or 5 hours after transfer $(b)$ and examined by transmission electron microscopy. Arrowheads represent tight junctions. Scale bar $=1 \mu \mathrm{m}$.

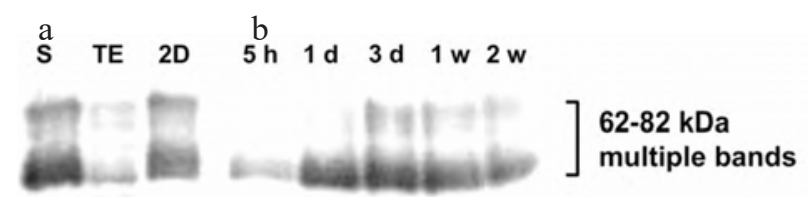

Fig. 4 Immunoblotting of harvested MDCK cells with anti-occludin antibody. (a) MDCK cells were plated on PIPAAm-grafted culture dishes at a cell density of $5 \times 10^{5}$ cells $/ 35 \mathrm{~mm}$ dish and cultured at $37^{\circ} \mathrm{C}$ for three weeks. Cells were recovered by physical scraping $(S)$ with rubber blades, trypsin-EDTA treatment (TE), or 2-D cell sheet manipulation (2D), and subjected to immunoblotting with anti-occludin antibody. $(b)$ TE-treated cells were replated at a confluent cell density and cultured for the indicated time. Cells were recovered by physical scraping and subjected to immunoblotting with anti-occludin antibody. 

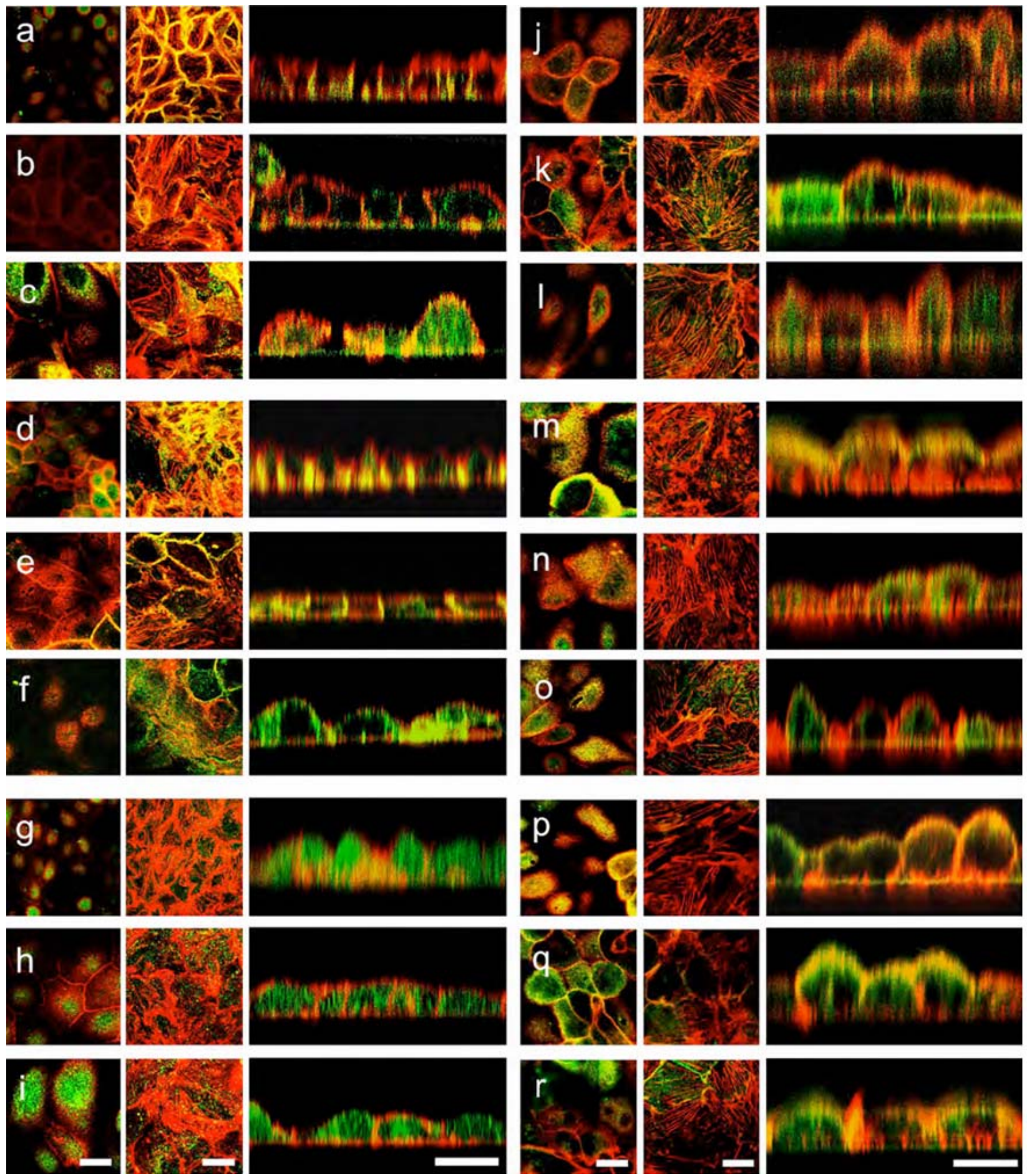

Fig. 5 Fluorescence microscopy of two-dimensionally manipulated MDCK or RPTEC cell sheets. Renal tubule cells cultured for three weeks at $37^{\circ} \mathrm{C}(a, d, g, j, m$ and $p)$ were subjected to two-dimensional manipulation $(b, e, h, k, n$ and $q$ ) or trypsin-treated subculture (c,f,i,l,o and $r$ ). After 5 hours (for MDCK cells, $a-i$ ) or 24 hours (for RPTECs, $j-r)$ reattached cells were fixed and double-stained with rhodamine-conjugated phalloidin for F-actin and anti$\mathrm{Na}^{+} / \mathrm{K}^{+}$-ATPase $(a-c)$, anti-GLUT-1 $(d-f)$, anti-SGLT-1 $(g-i)$, anti-AQP-1 $(j-l)$, anti-NEP $(m-o)$, or anti-DPP-IV ( $p$ $r$ ) antibody. These double-stained cells were observed under a confocal laser scanning microscope. Each set of microphotograph comprises three images (left: an apical portion of cell sheets, center: a basal portion of cell sheets, right: a vertical section image by computerized tomography). Images of F-actin (shown in red) and each antigen (shown in green) were superimposed. Co-localization of F-actin and each antigen are seen in yellow. Bars $=20 \mu \mathrm{m}$. 
plating and maintained throughout culture (Fig. 1). TJs thus developed three days after the initial seeding of MDCK cells and were maintained for over three weeks.

After culture for three weeks, confluent cell monolayers which had developed tight junctions were subjected to 2D cell sheet manipulation (Fig. 2). Confluent renal epithelial cell sheets were harvested together with the PVDF membranes, and no cell remnants were observed on PIPAAm-grafted culture dishes (Fig. 2b,e). On nongrafted TCPS dishes such cell sheet harvest could not be achieved and all cells remained on dish surfaces (data not shown). The PVDF membranes as well as the harvested MDCK or RPTEC cell sheets were then transferred onto porous membranes of culture inserts for transmission electron microscopic analysis or to collagencoated dishes. After 30 -min incubation at $20^{\circ} \mathrm{C}$, fresh culture medium was added and the PVDF membrane was removed from the cell sheets. Even after the removal of the PVDF membranes, the underlying renal tubule epithelial cell sheets still adhered to the new culture surfaces (Fig. 2c,f), with harvested cells adhering equally well to the porous membranes and collagen-coated dishes (data not shown).

MDCK cell sheets were then examined by transmission electron microscopy immediately (Fig. 3a) and 5 hours (Fig. 3b) after transfer from PIPAAm-grafted dishes to porous PET membranes, using 2-D manipulation. It was confirmed that MDCK cell sheets adhered closely to the PET membranes and that the transfer process was successful. Numerous microvilli and TJs (arrowheads) were observed at the apical and lateral membranes of the 2-D manipulated cells, respectively, demonstrating that transferred MDCK cells were able to maintain both their differentiated phenotypes and apical-basal polarity during the transfer process.

For biochemical analysis, MDCK cells were recovered by three methods; physical scraping with rubber blades, TE treatment, and the 2-D manipulation. Cell lysates were subjected to immunoblotting with an anti-occludin antibody (Fig. 4). Results showed that intact occludins were detected (resolved as several bands between 62 and 82 $\mathrm{kDa}$ ) in cell lysates obtained by the 2-D manipulation and physical scraping methods, but that TE treatment disrupted intact occuldins. TE treated cells were then replated under confluent conditions, and cultured at $37^{\circ} \mathrm{C}$. TE subcultured cells were recovered by physical scraping at the indicated time periods and subjected to immunoblotting (Fig. 4). The higher MW bands representing highly phosphorylated occludins were faintly detected after one-day, and further culture was required to regain occludin concentrations equal to those before cell harvest.

We finally examined the apical-basal polarity of renal tubule cells 5 hours after 2-D manipulation, by comparing the distributions of the $\mathrm{Na}^{+} / \mathrm{K}^{+}$-ATPase; two glucose transporters, GLUT-1 and SGLT-1; aquaporin-1 (AQP-1); and two brush border hydrolases, NEP and DPP-IV, in the 2-D manipulated or TE subcultured renal tubule epithelial cells. Cells were double-stained with rhodamineconjugated phalloidin for $\mathrm{F}$-actin and the antibodies against the indicated proteins and observed under a confocal laser scanning microscope (Fig. 5). $\mathrm{Na}^{+} / \mathrm{K}^{+}$-ATPase, which is responsible for ATP-dependent transport of $\mathrm{Na}^{+}$and $\mathrm{K}^{+}$ across cell membranes, and the facilitative glucose transporter GLUT-1 are known to exist at the basolateral membranes of renal proximal tubule cells (Cramer et al., 1992). These proteins were basolaterally distributed in both MDCK cells cultured for three weeks (Fig. 5a,d) and cells that underwent 2-D manipulation (Fig. 5b,e). Conversely, the $\mathrm{Na}^{+} / \mathrm{K}^{+}$-ATPase was localized in the cytoplasm (Fig. 5c) and GLUT-1 was detected on both the apical and basolateral sides in cells that had received TE treatment (Fig. 5f). It has been reported that SGLT-1, an isoform of the $\mathrm{Na}^{+}$-dependent glucose transporter, is localized at the apical plasma membranes in the epithelial cells of the small intestine (Hwang et al., 1991) and the kidney (Takata et al., 1991). In MDCK cells, however, SGLT-1 was observed in the cytoplasm before cell transfer (Fig. 5g). This distribution was retained even in both 2-D manipulated and TE subcultured cells (Fig. 5h and i, respectively). AQP-1, which functions as a water channel is abundantly present in kidney proximal tubules and almost exclusively localized in the plasma membranes (Elkjaer et al., 1995). There were no differences observed in the distribution of AQP-1 between RPTECs before transfer, after 2-D manipulation, or after TE subculture (Fig. 5j,k, and 1). NEP and DPP-IV are expressed on the apical membranes of differentiated renal proximal tubule cells (Tauc et al., 1989; Ronco et al., 1990; Jalal et al., 1991) and in our experiments were localized to the apical membranes of three-week cultured RPTECs (Fig. 5m and $\mathrm{p}$, respectively). The apical distribution of NEP was also observed in the 2-D manipulated RPTECs (Fig. 5n), but not in TE subcultured cells (Fig. 5o). DPP-IV however, was localized at the apical membranes in 2-D manipulated cells (Fig. 5q), and even in TE subcultured cells (Fig. 5r). As shown in Figure 5, the basolateral proteins, GLUT-1 and $\mathrm{Na}^{+} / \mathrm{K}^{+}$-ATPase, were not localized in a polarized fashion in TE subcultured renal tubule epithelial cells since TJs were not fully developed 5 hours after re-plating. It has been previously reported that an apical membrane protein can quickly become restricted to the apical areas upon contact with its substrate, even in the absence of tight junctions. However, under the same conditions a basolateral marker remains unpolarized (Vega-Salas et al., 1987).

In conclusion, trypsin digestion significantly hampered apical-basal cell polarity of epithelial cells, while cell polarity was maintained during temperature-responsive cell sheet harvest and transfer. Recently, we reported the early outcomes of clinical application of epithelial cell sheet transplantation to treat patients with total limbal epithelial stem cell deficiency (Nishida et al., 2004b). Epithelial cell sheets recovered from temperature-responsive culture surfaces, immediately showed barrier functions after transplantation onto the corneal stroma. This observed barrier function was also confirmed by the present study.

\section{Acknowledgements}

We appreciate the useful comments and technical criticism from Mr. Joseph Yang (Tokyo Women's Medical 
University). This work is supported in part by Grants-inAid for Scientific Research (16200036, and 16300161), the High-Tech Research Center Program, and the Center of Excellence Program for the 21st Century from the Ministry of Education, Culture, Sports, Science, and Technology in Japan and by the Core Research for Evolution Science and Technology from the Japan Science and Technology Agency.

\section{References}

Cramer SC, Pardridge WM, Hirayama BA, Wright EM (1992) Colocalization of GLUT2 glucose transporter, sodium/glucose cotransporter, and $\gamma$-glutamyl transpeptidase in rat kidney with double-peroxidase immunocytochemistry. Diabetes 41: 766-770.

Elkjaer ML, Birn H, Agre P, Christensen EI, Nielsen S (1995) Effects of microtubule disruption on endocytosis, membrane recycling and polarized distribution of Aquaporin-1 and gp330 in proximal tubule cells. Eur J Cell Biol 67: 57-72.

Heskins M, Guillent JE (1968) Solution properties of poly ( $N$-isopropylacrylamide). J Macromol Sci, Chem A2: 1441-1455.

Hirose M, Kwon OH, Yamato M, Kikuchi A, Okano T (2000) Creation of designed shape cell sheets that are noninvasively harvested and moved onto another surface. Biomacromolecules 1: 377-381.

Hwang ES, Hirayama BA, Wright EM (1991) Distribution of the SGLT1 Na+/glucose cotransporter and mRNA along the crypt-villus axis of rabbit small intestine. Biochem Biophys Res Commun 181: 1208-1217.

Jalal F, Lemay G, Zollinger M, Berteloot A, Boileau G, Crine P (1991) Neutral endopeptidase, a major brush border protein of the kidney proximal nephron, is directly targeted to the apical domain when expressed in MadinDarby canine kidney cells. J Biol Chem 266: 19826-19832.

Kushida A, Yamato M, Konno C, Kikuchi A, Sakurai Y, Okano T (1999) Decrease in culture temperature releases monolayer endothelial cell sheets together with deposited fibronectin matrix from temperature-responsive culture surfaces. J Biomed Mater Res 45: 355-362.

Kushida A, Yamato M, Konno C, Kikuchi A, Sakurai Y, Okano T (2000) Temperature-responsive culture dishes allow non-enzymatic harvest of differentiated MadinDarby canine kidney (MDCK) cell sheets. J Biomed Mater Res 51: 216-223

Kushida A, Yamato M, Kikuchi A, Okano T (2001) Two-dimensional manipulation of differentiated MadinDarby canine kidney (MDCK) cell sheets: the noninvasive harvest from temperature-responsive culture dishes and transfer to other surfaces. J Biomed Mater Res 54: 37-46.

Laemmli UK (1970) Cleavage of structural proteins during the assembly of the head of bacteriophage T4. Nature 227: 680-685.

Nandkumar MA, Yamato M, Kushida A, Konno C, Hirose M, Kikuchi A, Okano T (2002) Two-dimensional cell sheet manipulation of heterotypically co-cultured lung cells utilizing temperature-responsive culture dishes results in long-term maintenance of differentiated epithelial cell functions. Biomaterials 23: 1121-1130.

Nishida K, Yamato M, Hayashida Y, Watanabe K, Maeda N, Watanabe H, Yamamoto K, Nagai S, Kikuchi A, Tano Y, Okano T (2004a) Functional bioengineered corneal epithelial sheet grafts from corneal stem cells expanded ex vivo on a temperature-responsive cell culture surface. Transplantation 77: 379-385.

Nishida K, Yamato M, Hayashida Y, Watanabe K, Yamamoto K, Adachi E, Nagai S, Kikuchi A, Maeda N, Watanabe H, Okano T, Tano Y (2004b) Corneal reconstruction with tissue-engineered cell sheets composed of autologous oral mucosal epithelium. N Engl J Med 351: 1187-96.

Okano T, Yamada N, Sakai H, Sakurai Y (1993) A novel recovery system for cultured cells using plasma-treated polystyrene dishes grafted with poly ( $N$ isopropylacrylamide). J Biomed Mater Res 27: 1243-1251.

Ronco P, Antoine M, Baudouin B, Geniteau-Legendre M, Lelongt B, Chatelet F, Verroust P, Vandewalle A (1990) Polarized membrane expression of brush-border hydrolases in primary cultures of kidney proximal tubular cells depends on cell differentiation and is induced by dexamethasone. J Cell Physiol 145: 222-237.

Sakakibara A, Furuse M, Saitou M, Ando-Akatsuka Y, Tsukita S (1997) Possible involvement of phosphorylation of occludin in tight junction formation. J Cell Biol 137: 1393-1401.

Shimizu T, Yamato M, Isoi Y, Akutsu T, Setomaru T, Abe K, Kikuchi A, Umezu M, Okano T (2002) Fabrication of pulsatile cardiac tissue grafts using a novel 3dimensional cell sheet manipulation technique and temperature-responsive cell culture surfaces. Circ Res 90: e40.

Shiroyanagi Y, Yamato M, Yamazaki Y, Toma H, Okano $\mathrm{T}$ (2004) Urothelium regeneration using viable cultured urothelial cell sheets grafted on demucosalized gastric flaps. BJU Int 93: 1069-1075.

Takata K, Kasahara T, Kasahara M, Ezaki O, Hirano $\mathrm{H}$ (1991) Localization of $\mathrm{Na}(+)$-dependent active type and erythrocyte/HepG2-type glucose transporters in rat kidney: immunofluorescence and immunogold study. J Histochem Cytochem 39: 287-298.

Tauc M, Merot J, Bidet M, Koechlin N, Gastineau M, Othmani L, Poujeol P (1989) Antigenic expression of aminopeptidase $M$, dipeptidyl-peptidase IV and endopeptidase by primary cultures from rabbit kidney proximal tubule. Histochemistry 91: 17-30.

Vega-Salas DE, Salas PJ, Gundersen D, RodriguezBoulan E (1987) Formation of the apical pole of epithelial (Madin-Darby canine kidney) cells: polarity of an apical protein is independent of tight junctions while segregation of a basolateral marker requires cell-cell interactions. J Cell Biol 104: 905-916.

Yamada N, Okano T, Sakai H, Karikusa F, Sawasaki Y, Sakurai Y (1990) Thermo-responsive polymeric surfaces; control of attachment and detachment of cultured cells. Makromol Chem, Rapid Commun 11: 571-576.

Yamato M, Utsumi M, Kushida A, Konno C, Kikuchi A, Okano T (2001) Thermo-responsive culture dishes 
allow the intact harvest of multilayered keratinocyte sheets without dispase by reducing temperature. Tissue Eng 7: 473-480.

\section{Discussion with Reviewers}

C McFarland: During 2D manipulation the cell sheet, initially attached to a rigid substrate, will undergo a rapid transition to a much less constrained geometry as it is detached from the surface. Do the authors have any information as to the effect of this sudden change in mechanical environment on the physical properties and biological function of the cell sheet?

Authors: Adherent cells generate centripetal traction forces, and the equilibrium between the pulling forces developed by the cytoskeletal dynamics and the tensile stress of extracellular matrix has been noted and previously described. By reducing the incubation temperature, cells are released from these equilibrium forces because of the hydration of PIPAAm chain, and are detached from temperature-responsive culture dishes. In the case of cardiomyocytes, this change in the mechanical environment causes an increase in the beating frequency of cell sheets. However, in cases of endothelial cells and epithelial cells which are anchorage-dependent cells, if cell sheets remain floating in the culture medium after detachment, cells undergo detachment-induced apoptosis, anoikis.

C McFarland: In Figure 4, the expression of occludin appears lower in the 2D sheets than on the physically scraped cells. Since the latter treatment would be expected to cause a greater level of disruption of cell-cell contacts, it would be helpful if the authors could comment on this apparent discrepancy.

Authors: For physical scraping, lysis buffer containing protease inhibitors is first added to cell layers and the cell lysates are collected from the culture surfaces with a rubber blade. Therefore, no disruption of cell-cell contacts is caused by physical scraping. In this method, the proteins of cell-cell contacts can thus be collected intact, without loss.

C McFarland: Grafting of PIPAAm will alter the surface chemistry of the culture substrate. In turn this will influence the nature of protein adsorption and subsequent cell attachment. Have the authors examined the nature of the proteins adsorbed to the substrates, and its subsequent effect on cell attachment? Similarly, have they investigated the effect of this process on selecting specific subpopulations from heterogenous mixtures of cells such as found in isolates from primary tissue?

Authors: In order to obtain cell attachment/detachment with our system, we have optimized the density of PIPAAm that is grafted onto the TCPS dishes. Under normal culture conditions at $37^{\circ} \mathrm{C}$, cells adhere, spread and grow on the PIPAAm-grafted dishes at the appropriate density, similarly to on ungrafted TCPS dishes. This suggests that protein adsorption on temperature-responsive dishes is also similar to on TCPS dishes. We have examined the fibronectin matrix deposition of endothelial cells during the culture. The deposition and accumulation of the matrix were essentially the same on the nongrafted surfaces. The appropriate density of PIPAAm depends on the cell type. Therefore it is possible to use these temperature-responsive surfaces to select specific sub-populations from heterogenous mixtures, but at the present time, we do not have any data.

Y Missirlis: The maintenance of cells at $20^{\circ} \mathrm{C}$ for one hour plus 30 more minutes is relatively long. Is it necessary? Are there basic metabolic changes in the cells?

Authors: We have examined the incubation time to successfully recover cell sheets from temperatureresponsive culture dishes. Therefore, this incubation time is dependent on the cell type. In the case of renal epithelial cells, incubation for one hour is the shortest time possible to harvest all cells as a contiguous monolayer sheet with the PVDF membranes. The additional 30-min incubation after transfer is required for developing the observed cellsubstrate adhesion. When the cells were incubated at $37^{\circ} \mathrm{C}$ instead of $20^{\circ} \mathrm{C}$, cell-to-cell junctions were disrupted and many large holes formed in the transferred cell sheets. Cells near these holes exhibited stretched morphologies, implying that contractile forces exerted by cytoskeleton are the main cause. On the contrary, incubation at $20^{\circ} \mathrm{C}$ resulted in no defects in the transferred MDCK cell sheets because their metabolic activity, especially contractile forces are reduced at low temperature. This is why it is necessary to incubate for one and half hours at $20^{\circ} \mathrm{C}$. The intracellular esterase activity of transferred MDCK cells were assessed with calcein AM and results showed that the basic metabolic activity was maintained even in the transferred cell sheets. 